\title{
An Interesting Case of Pancreatic Tuberculosis Mimicking Pancreatic Carcinoma
}

\author{
Shanker Kundumadam*1, Hansini Laharwani ${ }^{2}$, Pradeep Kathi Reddy ${ }^{1}$ and Gokul Das ${ }^{3}$ \\ ${ }^{1}$ Wayne State University, Detroit, Michigan USA \\ ${ }^{2}$ University of Mississippi, Jackson, Mississippi USA \\ ${ }^{3}$ Post Graduate Institute of Medical Education and Research, Chandigarh India
}

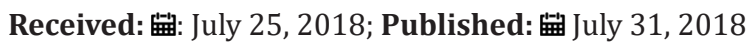

*Corresponding author: Shanker Kundumadam, 4201 St. Antoine street, 2E, Detroit, MI, 48201, Wayne State University/Detroit Medical Center, USA

\section{Introduction}

Pancreatic Tuberculosis is a rare entity. Here we present an interesting case of pancreatic tuberculosis which was initially falsely diagnosed as pancreatic cancer due to the imaging characteristics. Tissue culture from the lesion later grew Mycobacterium Tuberculosis thus diagnosing Tuberculus Pancreatitis.

\section{Case Report}

A 41-year-old female came to the clinic with a chief complaint of pain in abdomen and later had nausea and vomiting for 2 weeks. There was no fever. The patient was initially seen in another hospital where she was diagnosed with pancreatic carcinoma and referred for further management. The only significant physical sign was abdominal tenderness from past 3 weeks. Investigations revealed C-Reactive protein $44 \mathrm{mg} / \mathrm{l}$, ESR of $90 \mathrm{~mm} / \mathrm{hr}$. On endoscopic ultrasound multiple lymph nodes were seen at celiac axis, porta hepatis, supra pancreatic region and at aorto-caval region. Largest lymph node was noted at porta hepatis which was measuring around $2.3 \times 2.1 \mathrm{~cm}$ in size, round in shape, hypoechoic in nature with well-defined borders. A small sub centrimetric lymph node was noted in the mediastinum. No evidence of calcification or necrosis was visible. Of note no definite mass was seen in the pancreatic parenchyma.

Fine needle aspiration biopsy was done of the peri pancreatic lymphnode. At this point suspiscion for pancreatic tuberculosis was high. Acid Fast Bacillus (AFB) stain done on the sample was negative, but the Polymerase Chain Reaction (PCR) test came back positive for Mycobacterium Tuberculosis. The biopsy specimen also revealed caseating granulomas further solidifying the diangnosis of Tuberculus pancreatitis. The patient was started on therapy for Mycobacterium Tuberculosis with appropriate medications. She symptomatically improved over the next 6 months and gained weight back to her baseline. She had a follow up imaging with CT scan a year after the initial presentation which showed complete resolution of the adenopathy. On further follow up 2 years after the initial presentation she continues to remain symptom free and in good health.

\section{Discussion}

Pancreatic tuberculosis is a rare entity. In literature there have been handfuls of cases of pancreatic tuberculosis reported. There is striking similarity in the presentation of pancreatic tuberculosis and pancreatic malignancy. First report of such a case was in 1944 [1]. The symptomatology of the patients can vary from abdominal discomfort, pain, weight loss and rarely obstructive jaundice. Either hematogenous spread or direct spread from the neighboring caseating lymphondes could result in pancreatic tuberculosis $[2,3]$. Feng Xie et al. [4] noted that pancreatic tuberculosis is more common in young patients, females. This paper also looked at the various clinical symptomatology and characteristics. This differential has to be kept high priority especially in patients coming from areas where tuberculosis is endemic. In any patient presenting with pancreatic mass, who is a high risk for tuberculosis, pancreatic tuberculosis should be kept in the differential.

Endoscopic ultrasound is a very useful modality to aid in diagnosis and tissue sampling for staining and culture. There have been case reports patients even on anti tuberculus therapy developing pancreatic tuberculosis [5]. Patients need to be quarantined while in the hospital. Treatment is usually based on guideline appropriate therapy for extra pulmonary tuberculosis. In the western world immune-suppressed patients would be considered high risk for pancreatic tuberculosis and pancreatic tuberculosis in general.

\section{Conclusion}

Pancreatic tuberculosis can closely mimic pancreatic cancer. This has to be suspected in patients presenting with pancreatic mass who are high risk for tuberculosis. The response to therapy is usually excellent but prolonged therapy is warranted. 


\section{References}

1. Auerbach $O$ (1944) Acute generalized miliary tuberculosis. Am J Pathol 20: 121-136.

2. Feng X, Ronnie TPP, Shu GW, Bie p, Dong JH, et al. (2003) Tuberculosis of pancreas and peripancreatic lymph nodes in immunocompetent patients: experience from China. World J Gastroenterol 9(6): 1361-1364

3. Yang, Yan Jia Xiao, Qin Liu, Mei Yang, Kai Liu, et al. (2014) Pancreatic Tuberculosis Mimicking Pancreatic Carcinoma during Anti-Tuberculosis

ISSN: 2574-1241

DOI: 10.26717/BJSTR.2018.07.001507

Shanker Kundumadam. Biomed J Sci \& Tech Res

(C) This work is licensed under Creative

Submission Link: https://biomedres.us/submit-manuscript.php
Therapy: A Case Report. World Journal of Clinical Cases WJCC 2.5: 167169.

4. Franco Paredes C, Leonard M, Jurado R, Blumberg HM, Smith RM, et al. (2002) Tuberculosis of the pancreas: report of two cases and review of the literature. Am J Med Sci 323(1): 54-58.

5. Stock KP, Riemann JF, Stadler W, Rösch W, (1981) Tuberculosis of the pancreas. Endoscopy 13(4): 178-80.

$\begin{array}{ll}\text { BIOMEDICAL } & \text { Assets of Publishing with us } \\ \text { RESEARCHES } & \text { - Global archiving of articles } \\ \text { - Immediate, unrestricted online access }\end{array}$

